\title{
Treatment of tibia avulsion fracture of posterior cruciate ligament with total arthroscopic internal fixation with adjustable double loop plate: a retrospective cohort study
}

\section{Fan Zhang}

Tongji Hospital, Tongji Medical College, Huazhong University of Science and Technology

\section{Yaping Ye}

Tongji Hospital, Tongji Medical College, Huazhong University of Science and Technology

Wu Yu

Tongji Hospital, Tongji Medical College, Huazhong University of Science and Technology

Dan Yin

Tongji Hospital, Tongji Medical College, Huazhong University of Science and Technology Kai Xu ( $\nabla$ atqf0x@163.com )

Tongji Hospital, Tongji Medical College, Huazhong University of Science and Technology

\section{Research Article}

Keywords: Posterior cruciate ligament, Avulsion fracture, Arthroscopy, adjustable double loop plate

Posted Date: February 19th, 2021

DOI: https://doi.org/10.21203/rs.3.rs-181316/v1

License: (c) (1) This work is licensed under a Creative Commons Attribution 4.0 International License. Read Full License

Version of Record: A version of this preprint was published at Injury on April 1st, 2022. See the published version at https://doi.org/10.1016/j.injury.2022.04.003. 


\section{Abstract}

Background We aimed to evaluate the effect of a surgery technique-total arthroscopic internal fixation with adjustable double loop plate in treatment of patients with tibia avulsion fracture of posterior cruciate ligament $(\mathrm{PCL})$.

Methods From April 2018 to January 2020, 26 patients with tibia avulsion fracture of PCL treated in the joint surgery of our hospital were enrolled in this retrospective cohort study. According to the way of operation, the patients were divided into the experimental group (receiving total arthroscopic internal fixation with an adjustable double loop plate) and the control group (receiving traditional open surgery). The knee joint X-ray, CT or magnetic resonance imaging (MRI) were used to evaluate the injury or the fixation effect.

Results There were no significant differences in baseline patient demographics between the two groups in terms of age, sex, the average time from injury to surgery, type of surgery, preoperative knee range of motion, Lysholm score, IKDC score and KT-1000 score $(P>0.05)$. The average operation time in the experimental group ( $59.5 \pm 10.6$ minutes) was significantly shorter than that in the control group (96.8 \pm 13.7 minutes) $(P<0.05)$. Postoperative re-examination of knee $C T$ and $X$-ray showed that the fracture fragments were well fixed in all patients. The fracture was all healed 2 months after the operation. Postoperative posterior drawer tests were negative in all patients at final follow-up. All patients regained a satisfactory knee range of motion and the flexion and extension function and were satisfied with the results of surgery and rehabilitation. There was significant difference $(P<0.05)$ in the Lysholm scores, the IKDC scores, the KT1000 scores, and the ROM between preoperative and postoperative values in the control group and the experimental group. However, there were no significant differences in these values between the two groups $(p>0.05)$.

Conclusions Total arthroscopic internal fixation with adjustable double loop plate is relatively simple, safe, minimally invasive, and reliable. After taking this operation and rehabilitation training, patients with tibia avulsion fracture of PCL can regain a satisfactory knee range of motion and the flexion and extension function.

\section{Background}

Posterior cruciate ligament (PCL) is one of the important structures to stabilize the knee joint, and PCL injury will affect the stability of the knee joint. The incidence of PCL injuries has been reported to range from $3 \%$ to $23 \%$ of all knee injuries [1-2]. The tibia avulsion fracture of PCL is a special type of PCL injury, which mostly occurs in Asian countries with a massive number of cyclists [3-5], such as India and China. The characteristic of this type of fracture is that the fracture fragment is pulled up by the PCL, which would cause the joint relaxation.

Conservative treatment may cause the loss of knee joint mobility and cause complications such as stiffness [6]. Therefore, surgical treatment is preferred clinically. In the past, open surgery through the 
posterior approach of the knee joint was mostly used in clinical practice, but the surgical trauma was large. Then, because of the numerous vascular nerve bundles in the popliteal fossa, the open surgical risk and the incidence of postoperative infection was high [7]. With the development of arthroscopic technology, arthroscopic fixation and reconstruction surgery is widely used. Currently, the commonly used fixation methods include hollow screw fixation [8], Kirschner pin [9], door pin [10], tie wire [11], various transosseous tunnel suture or direct suture [12-18], steel plate and so on [19-20]. However, arthroscopic surgery is difficult and the fixation effect is potentially unstable.

The adjustable double loop plate used in our study is like an " $\nabla$ " shape at the fracture end and the outside of the tibia to clamp the root of the posterior cruciate ligament. The contact area between the plate and the fracture is large, which can provide uniform pressure distribution for the fracture. In this retrospective study, we aimed to evaluate the effect of a surgery technique-total arthroscopic internal fixation with adjustable double loop plate in treatment of patients with tibia avulsion fracture of PCL.

\section{Materials And Methods}

\section{Patients}

From April 2018 to January 2020, 26 patients with a tibia avulsion fracture of PCL treated in the joint surgery of our hospital were enrolled in this retrospective cohort study.

The inclusion criterion included: (1) The examinations by X-ray, CT scan, and magnetic resonance imaging (MRI) showed tibia avulsion fracture of PCL (Fig.1); (2) Time from injury to operation was less than 3 weeks; (3) With or without meniscus or collateral ligament injury, the displacement of the bone fragment was more than $5 \mathrm{~mm}$; (4) Posterior instability of knee joint was more than grade II. The exclusion criteria included: (1) involving peripheral tibial plateau fractures; (2) patients aged over 50 years with severe knee osteoarthritis; (3) patients with posterolateral complex injury; (4) patients with anterior cruciate ligament injury requiring reconstruction.

According to the way of operation, the patients were divided into the experimental group and the control group. Patients in the experimental group received total arthroscopic internal fixation with an adjustable double loop plate, while patients in the control group received traditional open surgery. All operations were performed by the same surgeon. All methods in the study were carried out in accordance with the Helsinki guidelines and declaration or any other relevant guidelines.

\section{Preoperative examination}

The knee range of motion (ROM) was checked and recorded. After anesthesia, the patients underwent posterior drawer examination at a $90^{\circ}$ position of the knee joint. The standard of the posterior drawer test was according to the displacement: less than $5 \mathrm{~mm}$ is grade $1 ; 5-10 \mathrm{~mm}$ is grade 2 ; more than $10 \mathrm{~mm}$ is grade 3. The preoperative knee joint X-ray, CT, and MRI were used to evaluate the injury. The Lysholm [21] 
knee joint score scale and International Knee Documentation Committee (IKDC) evaluation table [22] were used to evaluate the knee joint function, and KT-1000 was used to measure the stability of the knee joint.

\section{Surgery methods}

For the experimental group, the patients received combined spinal-epidural anesthesia with the supine position. A pneumatic tourniquet was used to stop bleeding at the base of the thigh. Arthroscopic anteromedial and anterolateral approaches were used to examine the articular cavity. The joint cavity was cleaned and the corresponding combined injuries, such as meniscus injury and free broken fracture block, were treated. The posterior compartment of the knee was penetrated from the outside to the inside, then the posterolateral and posteromedial approaches were established (Fig. 2A). The lens was turned to the posterolateral approach, and the posterior compartment synovium was cleaned with a planer through the posteromedial approach. The PCL insertion and fracture blocks were exposed (Fig. 2B), and the fracture blocks were replaced.

The traditional PCL tibial locator inserted through the anteromedial approach was used to locate the center of the bone mass (Fig. 2C), and the $2.0 \mathrm{~mm}$ Kirschner wire penetrated through the center of the fracture block (Fig. 2D). Under the guidance of Kirschner wire, the diameter of the bone tunnel was enlarged to $4.5 \mathrm{~mm}$ by hollow drill (Fig. 2E). The length of the tibial canal was measured. The adjustable loop plate and the fixed loop plate were connected in series to form a double plate system. The distance was from the length of the tibial tunnel plus $1.5 \sim 2 \mathrm{~cm}$ (Fig. 2F). The adjustable line was located at the outer entrance of the tunnel. The fixation loop was pulled into the rear compartment through the traction line (Fig. 2G). The adjustable loop was tightened outside the tunnel until the plate of the inner loop of the tunnel stabilized the PCL bone block on the tibial fracture surface (Fig. $2 \mathrm{H})$. The main operation was completed and the operation time was recorded.

For the experimental group, the patients received subarachnoid block anesthesia with a prone position, and the affected limb was bent $15^{\circ}$ to $20^{\circ}$ of the knee. A 3-4 cm long longitudinal incision was made under the transverse striation of the popliteal fossa posteriorly. The subcutaneous tissue and deep fascia were separated layer by layer. The space between the medial head and the lateral head of gastrocnemius muscle was exposed and blunt separation was carried out along this space. One hook was used to pull the lateral head of gastrocnemius muscle and popliteal fossa blood vessels and nerves, another hook was used to pull the medial head of gastrocnemius muscle to the medial side. When the exposure was sufficient, the posterior joint capsule was cut longitudinally. Then the fracture block was exposed and freshened. The avulsion fracture block was reduced. One or two Kirschner wires were used for temporary fixation perpendicular to the fracture line. After the C-arm X-ray fluoroscopy confirmed that the fracture reduction was satisfactory, 1-2 $4.0 \mathrm{~mm}$ cannulated screws were screwed in along the direction of Kirschner wire (Fig. 3).

\section{Postoperative examination and treatment}


After the operation, the patients underwent the posterior drawer test. On the second day after the operation, the drainage tube was removed, and the patient received straight leg raising and patellar pushing training (Fig. 4). The X-ray and CT examinations were taken within one week after the operation to evaluate the fixation effect. The lower limbs were fixed in $0{ }^{\circ}$ position with an adjustable brace for 2 weeks. After that, the flexion degree increased by $15^{\circ}$ every week until $90^{\circ}$ flexion in the eighth week. From the 5th week to the 8th week after the operation, the weight-bearing of the affected limb increased gradually. After fracture healing, complete weight-bearing was feasible. At the last follow-up, the Lysholm scale and IKDC evaluation table were used to evaluate the knee joint function, and the KT-1000 was used to measure the stability of the knee joint.

\section{Statistical analysis}

All the data collected in this study were analyzed by SPSS 23.0 software. Normally distributed measurement data were expressed as mean \pm standard deviation (SD), while non-normally distributed measurement data were expressed as median (interquartile range), and the comparisons were examined by Student t-test (between the two groups) or paired t-test (between the pre-and post-operation) and Mann-Whitney test (non-parametric distribution). The categorical data were expressed as $n(\%)$, and the differences between the two groups were examined by chi-square analysis. $\mathrm{P} \otimes 0.05$ was considered statistically significant.

\section{Results}

\section{General data of patients}

A total of 26 patients were enrolled in this study, with an average age of 36.2 years (from 19 to 54), including 19 males and 7 females. There were 12 cases of traffic injury and 14 cases of sports injury. The average time from injury to surgery was 12 days (from 7 to 24 days). Preoperative posterior drawer tests were positive in all patients before surgery, among them, there were 17 patients with grade 2 posterior laxity and 9 patients with grade 3 posterior laxity. Two patients had a longitudinal tear of the posterior horn of the medial meniscus and body, and three patients had partial injury of the medial collateral ligament. The mean follow-up time after surgery was 12.4 months (from 6 months to 19 months). There were no significant differences in baseline patient demographics between the two groups in terms of age, sex, the average time from injury to surgery, and type of surgery $(P>0.05)($ Table 1$)$.

Table 1. General information of patients. 


\begin{tabular}{|c|c|c|c|}
\hline & $\begin{array}{c}\text { Control group } \\
\mathrm{n}=13\end{array}$ & $\begin{array}{c}\text { Experimental group } \\
\mathrm{n}=13\end{array}$ & $\mathrm{P}$ \\
\hline Age (years) & 38 & 36 & 0.248 \\
\hline \multicolumn{4}{|l|}{ Gender } \\
\hline Male & 9 & 10 & 0.936 \\
\hline Female & 4 & 3 & 0.940 \\
\hline Average time from injury to surgery $\llbracket$ days $\square$ & 13.5 & 12.6 & 0.185 \\
\hline \multicolumn{4}{|l|}{ Type of injury } \\
\hline Traffic injury & 5 & 7 & 0.241 \\
\hline Sports injury & 8 & 6 & 0.266 \\
\hline \multicolumn{4}{|l|}{ Preoperative posterior drawer tests } \\
\hline grade 2 posterior laxity & 9 & 8 & 0.835 \\
\hline grade 3 posterior laxity & 4 & 5 & 0.862 \\
\hline
\end{tabular}

There were no differences in knee range of motion, Lysholm score, IKDC score, and KT-1000 score between the two groups before surgery.

There were no differences between the experimental group and the control group in IKDC score $\$ 54.1 \pm 6.2$ vs $53.2 \pm 7.1 \rrbracket \mathrm{P}=0.812 \rrbracket$, Lysholm score $\varangle 37.5 \pm 4.1$ vs $36.8 \pm 2.5 \bigotimes \mathrm{P}=0.636 \rrbracket, \mathrm{KT}-1000$ score $\varangle 9.8 \pm 0.6 \mathrm{~mm}$ vs $9.6 \pm 0.4 \mathrm{~mm} \otimes \mathrm{P}=0.401 \rrbracket$, and $\mathrm{ROM} \otimes 30 \pm 4.5^{\circ}$ vs $31 \pm 3.7^{\circ} \otimes \mathrm{P}=0.723 \rrbracket$ before surgery (Table 2.).

Table 2. Comparison of knee range of motion, Lysholm score, IKDC score and KT-1000 score between the experimental group and the control group pre- and post- operation.

\begin{tabular}{clccc}
\hline \multirow{2}{*}{ Time } & Variable & Control group & Experimental group & P \\
& & & & \\
& & & & \\
\hline Preoperative & Knee range of motion & $31 \pm 3.7^{\circ}$ & $30 \pm 4.5^{\circ}$ & 0.723 \\
& Lysholm score & $36.8 \pm 2.5$ & $37.5 \pm 4.1$ & 0.636 \\
& IKDC score & $53.2 \pm 7.1$ & $54.1 \pm 6.2$ & 0.812 \\
& KT-1000 score (mm) & $9.6 \pm 0.4$ & $9.8 \pm 0.6$ & 0.401 \\
Final follow-up & Knee range of motion & $131 \pm 4.2^{\circ}$ & $133.5 \pm 6.3^{\circ}$ & 0.820 \\
& Lysholm score & $81.5 \pm 3.2$ & $84.3 \pm 5.2$ & 0.915 \\
& IKDC score & $83.6 \pm 3.7$ & $84.5 \pm 5.1$ & 0.799 \\
& KT-1000 score & $1.7 \pm 0.5$ & $1.6 \pm 0.4$ & 0.827 \\
\hline
\end{tabular}

IKDC: International Knee Documentation Committee.

The operation time of the experimental group was less than that of the control group.

The average operation time in the experimental group was $59.5+10.6$ minutes, which was significantly shorter than that in the control group $(96.8+13.7$ minutes $)(P<0.05)$. In the control group, one patient suffered from an intraoperative collateral ligament injury and underwent primary collateral ligament repair.

All patients had good outcomes postoperatively. 
Postoperative re-examination of knee CT and X-ray showed that the fracture fragments were well fixed in the control group (Fig. 3) and experimental group (Fig. 5). Postoperative drawer tests were negative in all patients at final follow-up. The fracture was all healed 2 months after the operation. All patients regained a satisfactory knee range of motion and the flexion and extension function (Fig. 6). And all patients were satisfied with the results of surgery and rehabilitation.

In the experimental group, the postoperative ROM $\left(133.5 \pm 6.3^{\circ}\right)$, Lysholm scores $(84.3 \pm 5.2)$, and IKDC scores (4.5 \pm 5.1$)$ increased, and the postoperative KT1000 scores (1.6 \pm 0.4$)$ dropped. There was a significant difference in the Lysholm scores $(P=0.001)$, the IKDC scores $(P=0.001)$, the KT1000 scores $(P=0.001)$, and the ROM $(P=0.000)$ between preoperative and postoperative values in the experimental group. (Table. 3)

Table 3. Comparison of knee range of motion, Lysholm score, IKDC score and KT-1000 score between pre- and post- operation.

\begin{tabular}{llcrc}
\hline & Variable & Preoperative & Final follow-up & P \\
& & & & \\
\hline Control group & Knee range of motion & $31 \pm 3.7^{\circ}$ & $131 \pm 4.2^{\circ}$ & 0.000 \\
& Lysholm score & $36.8 \pm 2.5$ & $81.5 \pm 3.2$ & 0.001 \\
& IKDC score & $53.2 \pm 7.1$ & $83.6 \pm 3.7$ & 0.001 \\
& KT-1000 score (mm) & $9.6 \pm 0.4$ & $1.7 \pm 0.5$ & 0.001 \\
Experimental group & Knee range of motion & $30 \pm 4.5^{\circ}$ & $133.5 \pm 6.3^{\circ}$ & 0.000 \\
& Lysholm score & $37.5 \pm 4.1$ & $84.3 \pm 5.2$ & 0.000 \\
& IKDC score & $54.1 \pm 6.2$ & $84.5 \pm 5.1$ & 0.001 \\
& KT-1000 score & $9.8 \pm 0.6$ & $1.6 \pm 0.4$ & 0.001 \\
\hline
\end{tabular}

IKDC: International Knee Documentation Committee.

In the control group, the postoperative ROM $\left(131 \pm 4.2^{\circ}\right)$, Lysholm scores (81.5 \pm 3.2$)$, and IKDC scores $(83.6 \pm 3.7)$ also increased, and the KT1000 scores (1.7 \pm 0.5$)$ dropped. There was a significant difference in the Lysholm scores $(P=0.001)$, IKDC scores $(P=0.001)$, KT1000 scores $(P=0.001)$, and the ROM $(P=0.000)$ between preoperative and postoperative values in the control group. (Table. 3 )

\section{There were no significant differences in knee range of motion, Lysholm score, IKDC score, and KT-1000 score between the two groups at the final follow-up.}

We then compared the postoperative ROM, Lysholm scores, IKDC scores, and KT1000 scores between the experimental group and the control group, and found that there were no significant differences in these values between the two groups $(P>0.05)$. (Table. 2)

\section{Discussion}

The incidence of tibia avulsion fracture of PCL is very low in clinic, and the clinical manifestation of tibia avulsion fracture of PCL is similar to that of PCL injury. The diagnosis of tibia avulsion fracture of PCL needs to be confirmed by imaging, and the knee joint X-ray, CT, and MRI are routinely required before surgery to determine the size and displacement of the fracture fragment. The fracture with large 
fragments and no displacement can be fixed with plaster, however, surgery is needed for the displaced fractures. Traditional surgical methods are mainly open reduction and internal fixation, and there are also reports of successful open surgical treatment combined with arthroscopy [23]. The emerging total arthroscopic treatment technology not only has a small incision (only $0.5 \mathrm{~cm}$ skin incision) but also can repair incidental injuries, such as meniscal injuries, which reduces the risk of postoperative infection and improve patient satisfaction [24-25]. In this retrospective study, we analyzed the preoperative and prognosis of patients with tibia avulsion fracture of PCL who underwent traditional open internal fixation or total arthroscopic internal fixation with adjustable double loop plate in our hospital, and found that the effect of total arthroscopic internal fixation with adjustable double loop plate was not inferior to that of traditional open internal fixation.

Hooper et al. [26] retrospective analyzed the literature on avulsion fractures of PCL from 1975 to 2016 and found that more patients who underwent arthroscopic surgery regained normal knee range of motion than patients who underwent traditional open surgery, and the IKDC scores in the patients underwent arthroscopic surgery were more than that of patients underwent the traditional open surgery. In our study, there were no significant differences in postoperative ROM and the IKDC scores between the experimental group and the control group, and all patients regained a satisfactory knee range of motion and flexion and extension function. In this total arthroscopic surgery, the Rigidloop ${ }^{\mathrm{TM}}$ adjustable system of Johnson \& Johnson was chosen because its adjustable loop can be fully tightened, making it more convenient to calculate the length of the reserved loop between two steel plates. And the key point of the operation was to use an adjustable double-loop steel plate in a " $\nabla$ " shape to clamp the root of the posterior cruciate ligament and provide a uniform pressure distribution for the fracture.

Screw fixation is the common fixation method for an avulsion fracture. In recent years, various highstrength suture fixation methods have been widely used and achieved good results [12-17]. The difficulties of various suture techniques are mainly the bundling and reduction of bone fracture fragments, the establishment of bone channels, and the completion of suture winding. Therefore, the suture technique is difficult and needs to be mastered skillfully. The fixation method in our surgery does not need suture and winding, and the operation process is simple and easy to grasp. This technique requires only one tunnel to fix the fracture, which avoids the operational difficulties caused by double tunnels and reduces the operation time. This technique also does not require a second surgical removal of the internal fixation. The operation time was not long, which would reduce the risk of infection and postoperative thrombosis.

The following points should be noted in this technique: The size of the fracture fragment is an important parameter, and the $4.5 \mathrm{~mm}$ tunnel may destroy the thinner fracture fragments. Therefore, we should evaluate the size of the fracture fragment in detail and avoid fracture comminution of the thinner fracture fragment by adjusting the position of the tibial positioner. This technique needs two loop plates, one of which is the adjustable loop plate, so the operation cost is high. Besides, for elderly patients with osteoporosis, it is necessary to further study whether it is suitable for using this surgical method. 


\section{Limitations}

There are still some limitations to our study. The follow-up period was relatively short. Long-term followup may provide a more comprehensive understanding of the effect of the two surgical techniques on long-term motor function. Our study was mainly conducted in our hospital, and it was a single-center study. The sample size of this study is small, and larger sample size will have more persuasion. Then, this study lacks comparison with other minimally invasive surgery methods.

\section{Conclusion}

Total arthroscopic internal fixation with adjustable double loop plate is relatively simple, safe, minimally invasive, and reliable. After taking this operation and rehabilitation training, patients with tibia avulsion fracture of PCL can regain a satisfactory knee range of motion and the flexion and extension function. The application of this surgical technique provides a new reference for the minimally invasive repair of intra-knee fractures and has great prospects for clinical promotion.

\section{Abbreviations}

posterior cruciate ligament $(\mathrm{PCL})$

magnetic resonance imaging (MRI)

range of motion (ROM)

International Knee Documentation Committee (IKDC)

Posterior cruciate ligament (PCL)

standard deviation (SD)

\section{Declarations}

Ethics approval and consent to participate: The study protocol was approved by the Ethics Committee of Tongji Hospital, Tongji Medical College, Huazhong University of Science and Technology. Written informed consent was obtained from all subjects

Consent to Publish: Not applicable.

Authors Contributions: FZ and KX contributed to the conception and design of the study; YPY, DY and WY performed the experiments, collected and analyzed data; FZ and KX wrote the manuscript; All authors reviewed and approved the final version of the manuscript.

Funding: No funding was received for this study. 
Competing Interests: The authors declare that they have no competing interests.

Availability of data and materials: The datasets generated during and analysed during the current study are not available due to the article unpublished but are available from the corresponding author on reasonable request

\section{Acknowledgements: None}

\section{References}

1. Fontbote' CA, Sell TC, Laudner KG, et al. Neuromuscular and biomechanical adaptations of patients with isolated deficiency of the posterior cruciate ligament. Am J Sports Med. 2005;33:982-989.

2. Veltri DM, Warren RF. Isolated and combined posterior cruciate ligament injuries. J Am Acad Orthop Surg. 1993;1:67-75.

3. Bali K, Prabhakar S, Saini U, Dhillon MS. Open reduction and internal fixation of isolated PCL fossa avulsion fractures. Knee Surg Sports Traumatol Arthrosc. 2012;20:315-321

4. Kim SJ, Shin SJ, Choi NH, Cho SK. Arthroscopically assisted treatment of avulsion fractures of the posterior cruciate ligament from the tibia. J Bone Joint Surg [Am] 2001;83-A:698-708.

5. Kim SJ, Shin SJ, Cho SK, Kim HK. Arthroscopic suture fixation for bony avulsion of the posterior cruciate ligament. Arthroscopy 2001;17:776-780.

6. Montgomery SR, Johnson JS, McAllister DR, Petrigliano FA. Surgical management of PCL injuries: indications, techniques, and outcomes. Curr Rev Musculoskelet Med. 2013;6:115-123.

7. Wajsfisz A囚Makridis KG『Van Den Steene JY. Fixation of posterior cruciate ligament avulsion fracture with the use of a suspensory fixation[J]. Knee Surg Sports Trauma-tol Arthrosc $2012 \rrbracket 20 \rrbracket 5 \rrbracket: 996-999$.

8. Chen W, Luo W, Chen Z, Jiang Y. Treatment of posterior cruciate ligament avulsion fractures of the tibia using a toothed plate and hollow lag screw. Singapore Med J. 2016;57:39-44.

9. McLennan JG. The role of arthroscopic surgery in the treatment of fractures of the intercondylar eminence of the tibia. J Bone Joint Surg Br 1982;64:477-80

10. Torisu T. Isolated avulsion fracture of the tibial attachment of the posterior cruciate ligament. J Bone Joint Surg Am 1977;59:68-72.

11. Calpur OU, Copuroglu C, Ozcan M. Avulsion fractures of both anterior and posterior cruciate ligament tibial insertions. Knee Surg Sports Traumatol Arthrosc 2002;10:223-5

12. L. B. Chen, H. Wang, K. Tie, A. Mohammed, Y. J. Qi Arthroscopic fixation of an avulsion fracture of the tibia involving the posterior cruciate ligament. Bone Joint J.2015;97-B:1220-5.

13. S.J. Rhee, et al., Arthroscopic reduction of posterior cruciate ligament tibial avulsion fracture using two cross-linked pull-out sutures: A surgical technique and case series. Injury. 2019 Mar;50(3):804810.

14. Boutsiadis A, Karataglis D, Agathangelidis F, Ditsios K, Papadopoulos P. Arthroscopic 4-point suture fixation of anterior cruciate ligament tibial avulsion fractures. Arthrosc Tech 2014;3:e683-7. 
15. Zhao J, He Y, Wang J. Arthroscopic treatment of acute tibial avulsion fracture of the posterior cruciate ligament with suture fixation technique through Y-shaped bone tunnels Arthroscopy 2006;22:172-81.

16. Verdano MA, Pellegrini A, Lunini E, Tonino P, Ceccarelli F. Arthroscopic absorbable suture fixation for tibial spine fractures. Arthrosc Tech 2014;3:e45-8.

17. Yoon JR, Park CD , Lee DH. Arthroscopic suture bridge fixation technique with multiple crossover ties for posterior cruciate ligament tibial avulsion fracture. Knee Surg Sports Traumatol Arthrosc. 2018 Mar;26(3):912-918

18. Gui J, Wang L, Jiang Y, Wang Q, Yu Z, Gu Q, et al. Singletunnel suture fixation of posterior cruciate ligament avulsion fracture. Arthroscopy 2009;25:78-85.

19. Wajsfisz A, Makridis KG, Van Den Steene JY, Djian P. Fixation of posterior cruciate ligament avulsion fracture with the use of a suspensory fixation. Knee Surg Sports Traumatol Arthrosc 2012;20:996999

20. Horas U, Meissner SA, Heiss C, Schnettler R. Arthroscopic fixation of posterior cruciate ligament avulsion fractures: A new minimally invasive technique. Knee Surg Sports Traumatol Arthrosc 2010;18:781-783.

21. Lysholm J, Gillquist J. Evaluation of knee ligament surgery results with special emphasis on use of a scoring scale. Am J Sports Med 1982;10:150-154.

22. Ebrahimzadeh $\mathrm{MH}$, Makhmalbaf $\mathrm{H}$, Golhasani-Keshtan F, et al. The International Knee Documentation Committee (IKDC) Subjective Short Form: a validity and reliability study. Knee Surgery Sports Traumatol Arthrosc, 2015, 23(11): 3163-3167.

23. Petrillo S, Volpi P, Papalia R, Maffulli N, Denaro V. Management of combined injuries of the posterior cruciate ligament and posterolateral corner of the knee: A systematic review. Br Med Bull 2017;123:47-57.

24. Bhattacharyya $\mathrm{M}$, Bradley $\mathrm{H}$. Intraoperative handling and wound healing of arthroscopic portal wounds: A clinical study comparing nylon suture with wound closure strips. J Perioper Pract 2008;18:194-196, 198

25. Badran MA, Elgeidi AE, Elganiny A, Sallab RME. Posterior cruciate ligament tibial avulsion fracture: A novel arthroscopic fixation technique versus open posteromedial fixation. Orthop Spine Sports Med 2017;1:002.

26. Hooper PO 3rd 1, Silko C 1, Malcolm TL 2, Farrow LD. Management of Posterior Cruciate Ligament Tibial Avulsion Injuries: A Systematic Review. Am J Sports Med. 2018 Mar;46(3):734-742.

\section{Figures}




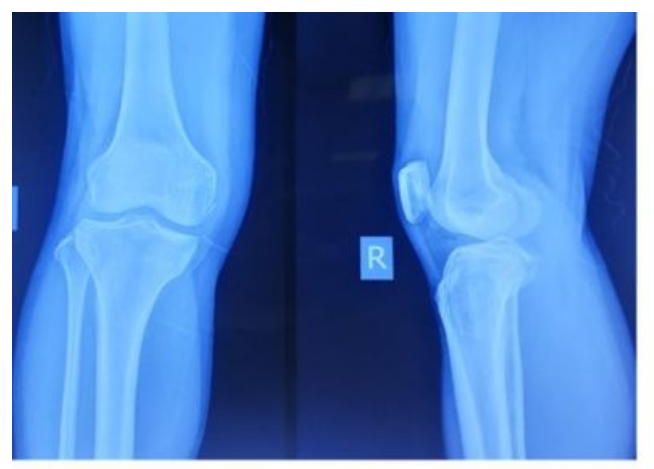

A

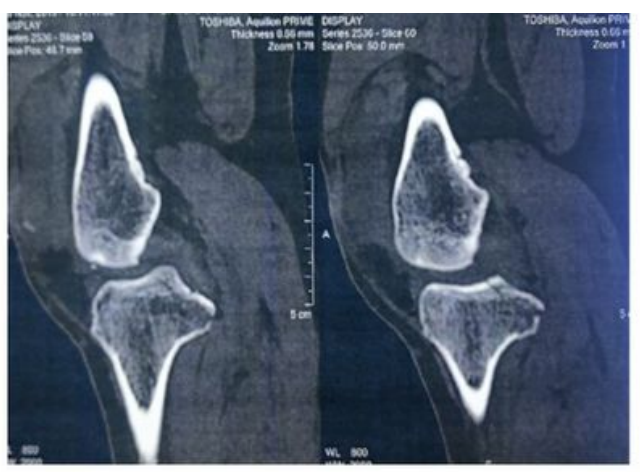

$\mathrm{B}$

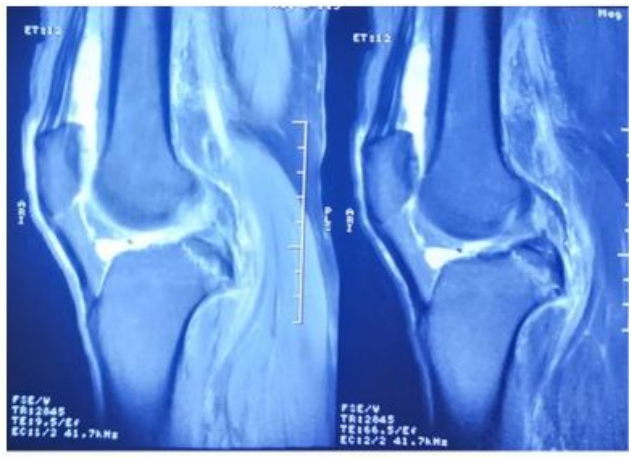

$\mathrm{C}$

\section{Figure 1}

Preoperative X-ray, CT scan, and MRI examinations. The preoperative examinations by X-ray (A), CT (B), and MRI (C) indicated the tibia avulsion fracture of posterior cruciate ligament.

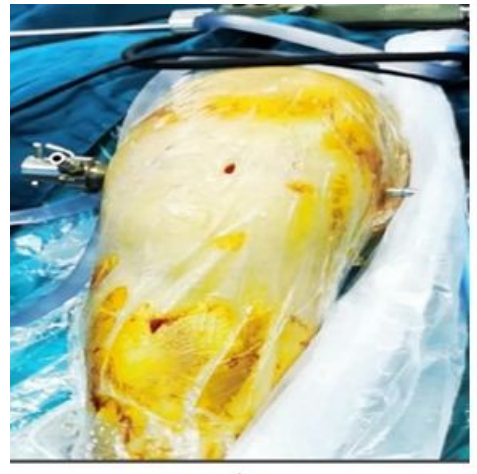

A

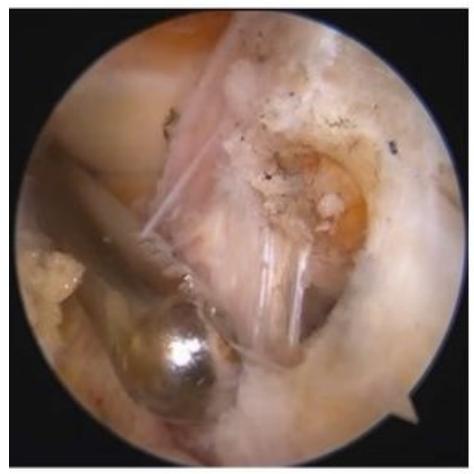

$\mathrm{E}$

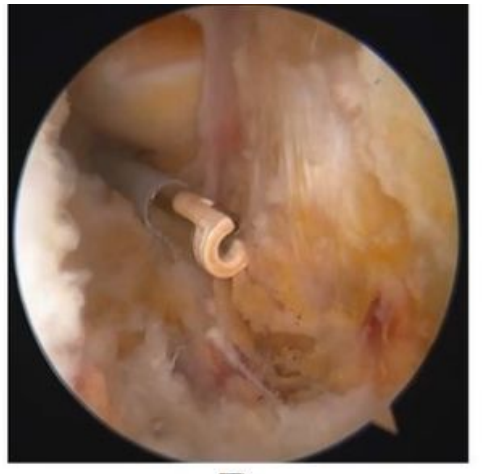

$\mathrm{B}$

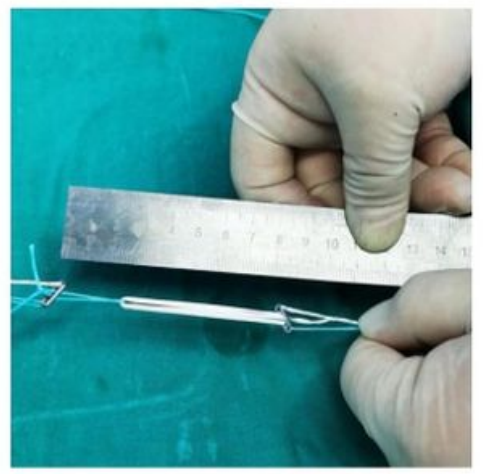

F

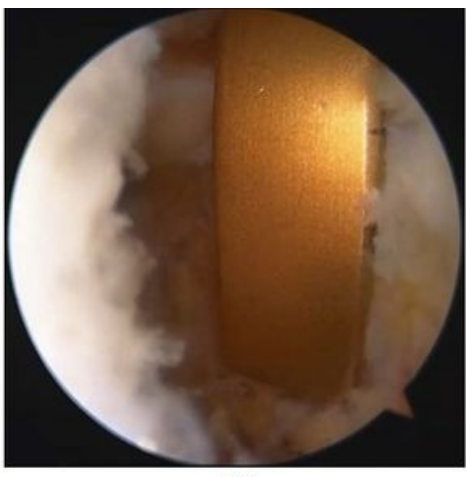

$\mathrm{C}$

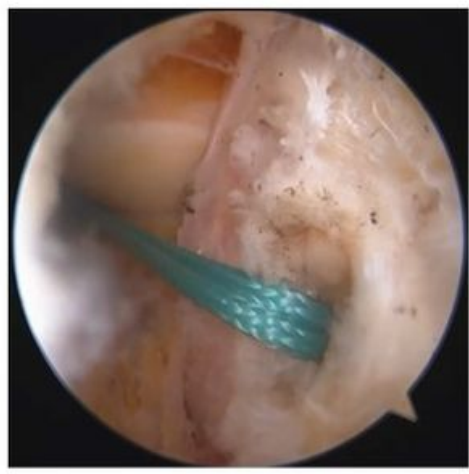

G

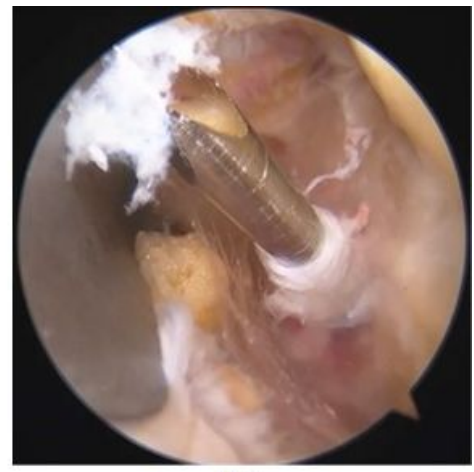

$\mathrm{D}$

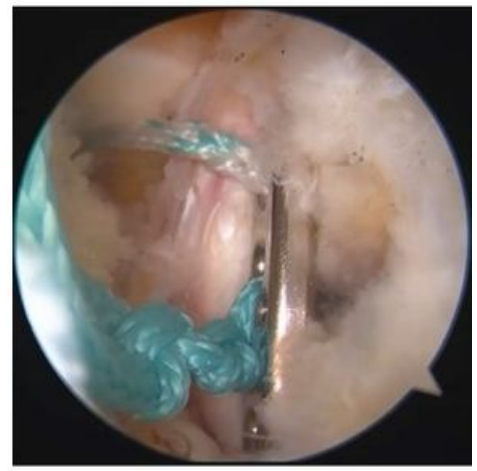

$\mathrm{H}$

\section{Figure 2}

The operation process of experimental group. A The posterior compartment of the knee was penetrated from the outside to the inside, B The PCL insertion and fracture blocks were exposed, C The traditional PCL tibial was used to locate the center of the bone mass, D The $2.0 \mathrm{~mm}$ Kirschner wire penetrated through the center of the fracture block, E The diameter of bone tunnel was enlarged to $4.5 \mathrm{~mm}$ by hollow drill, F Measured the length of tibial canal and formed a double plate system, $G$. The fixation loop was 
pulled into the rear compartment through the traction line, $\mathrm{H}$ Stabilized the PCL bone block on the tibial fracture surface.

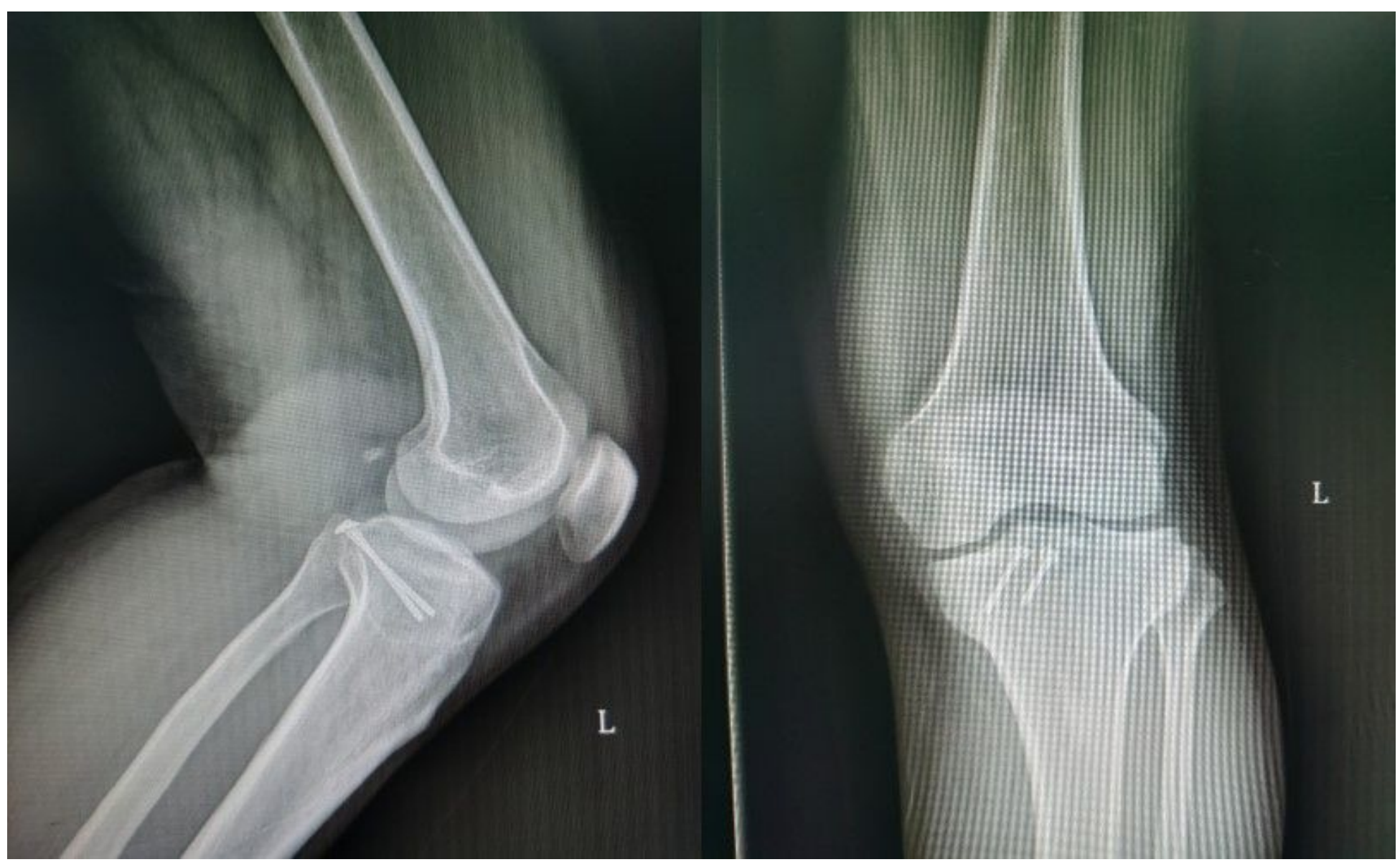

\section{Figure 3}

X-ray results of traditional surgery: fixation of the tibia avulsion fracture of posterior cruciate ligament with two cannulated screws.
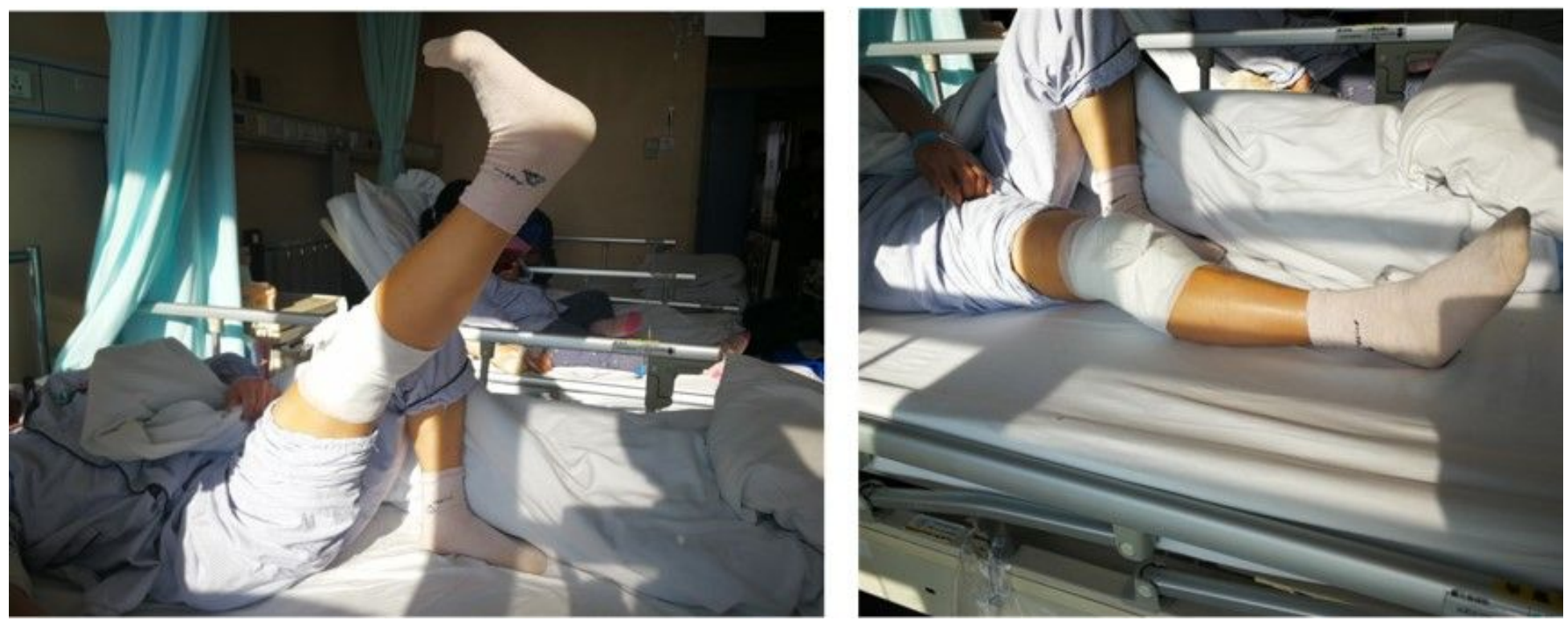

Figure 4 
On the second day after the operation, the drainage tube was removed, and the patient received straight leg raising and patellar pushing training.

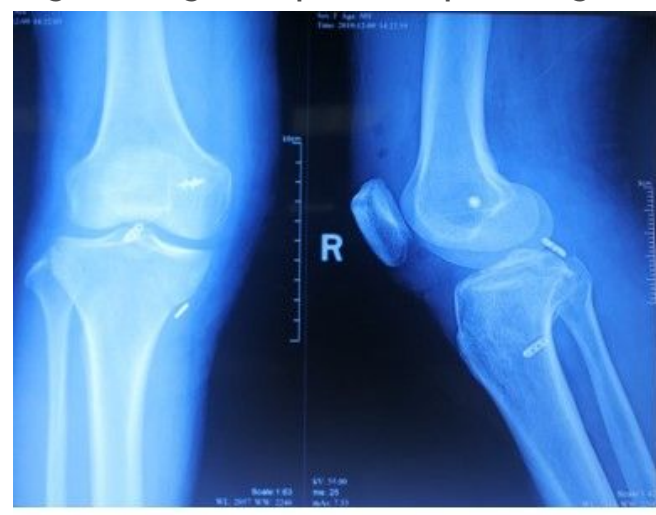

A

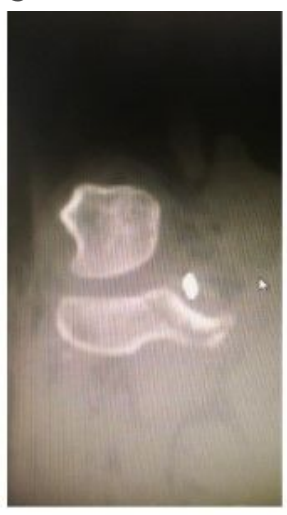

$\mathrm{B}$
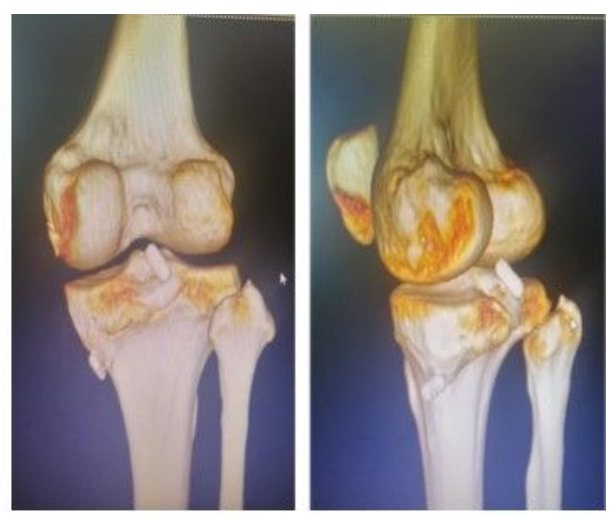

$\mathrm{C}$

\section{Figure 5}

Imaging examination in experimental group 5 days after operation. A Postoperative $\mathrm{X}$-ray positive and lateral radiographs showing plate position, $\mathrm{B}$ Postoperative $\mathrm{CT}$ showing the relative position of the plate and fragment, C Postoperative three-dimensional CT showing plate position.
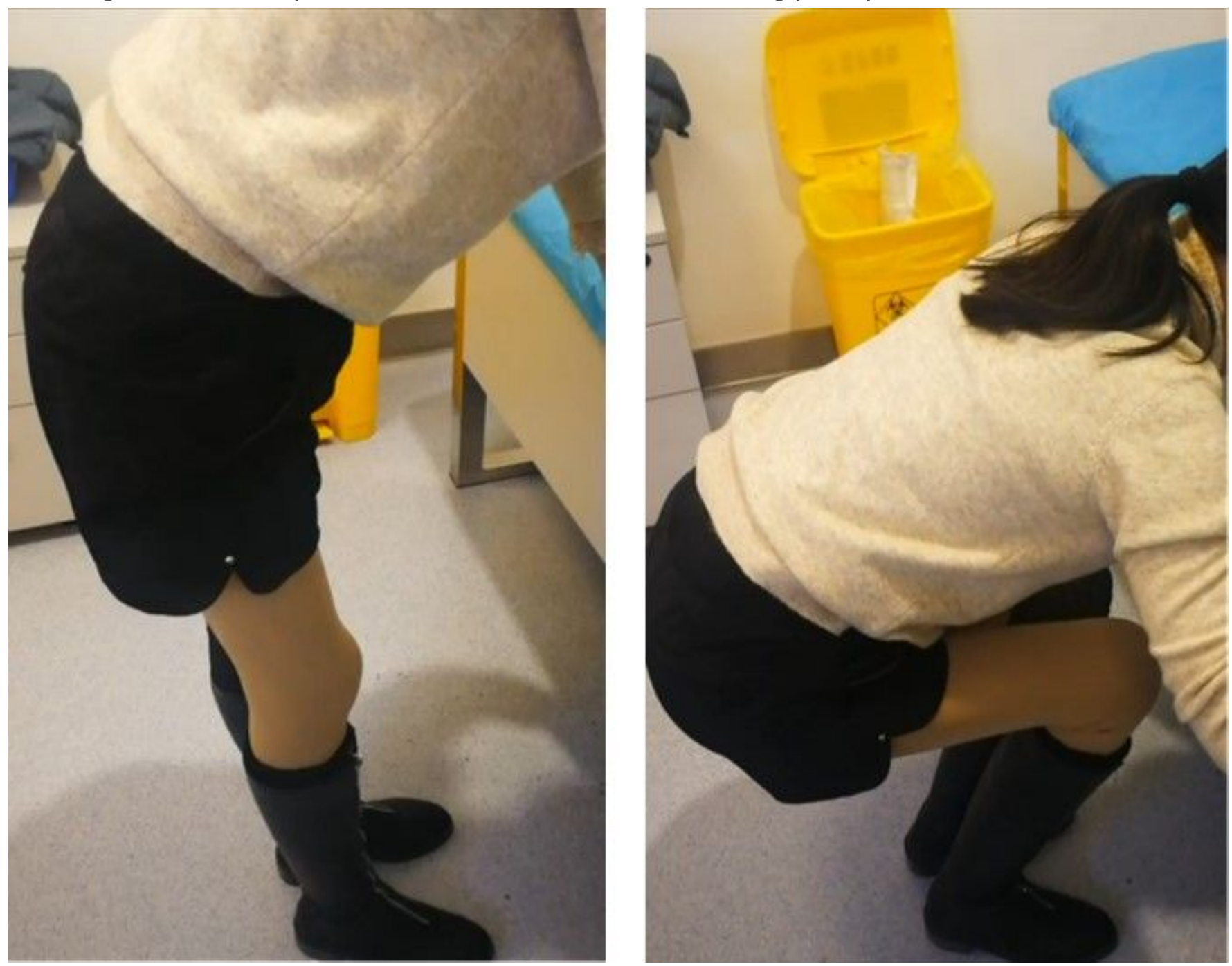
Figure 6

Extension and flexion of knee joint three months after operation. 